\title{
Gut
}

Leading article

\section{Chemotherapy for gastric cancer}

In the United Kingdom, gastric carcinoma is the sixth most common malignancy; recent reports showed that there were 10653 cases and 9714 deaths in 1986.' There is a considerable worldwide variation in the incidence, with age standardised rates varying from 75:100 000 per year in men in Osaka, Japan to 11:100000 per year in Connecticut, USA. In economically developed countries the incidence of gastric cancer has declined over the past 20 years. The reasons for this demographic trend are unclear but important factors include changing diet, improved methods of food preservation, the food preservatives themselves, and decreased exposure to environmental carcinogens. Recent studies have shown that the risk of developing gastric cancer can be reduced by eating green and yellow vegetables frequently, perhaps because vitamins $A$ and $C$ may give potential protection from carcinogens. ${ }^{2}$

Because the early course of the disease is often silent, most patients present with advanced unresectable disease. Two thirds of patients are more than 60 years of age. Both these factors contribute to the extremely poor prognosis at presentation. The median survival of the untreated patient with gastric cancer at presentation is four months and this increases to six months for those undergoing palliative resection. Although surgery is cited as the main hope for cure, only $7 \%$ of patients treated by radical surgery survive five years and the median survival of these patients is only eight months. Radiotherapy is an ineffective local treatment for gastric cancer because of poor tissue tolerance to potentially curative radiation dosage regimens.

Gastric carcinoma is a chemosensitive tumour and early reports showed response rates of over $20 \%$ for adriamycin, cisplatin, 5-fluorouracil, and mitomycin $\mathrm{C}$ given as single agents. ${ }^{3}$ This led to their inclusion in numerous combination chemotherapy programmes. Interpretation of the relative benefits of these combinations is difficult because of the publication bias for positive reporting, the limited numbers of patients treated, and the inherent difficulty of accurately assessing response. In 1977, radically improved response rates were reported for the 5-fluorouracil, adriamycin, and mitomycin-C (FAM) regimen with remissions of $42 \%$ in a group of 62 patients. ${ }^{4}$ The Gastrointestinal Cancer Study Group carried out a number of randomised trials comparing FAM with other combination chemotherapy regimens. ${ }^{5-7}$ Although in these studies the level of activity was not as high as originally reported, subsequent work by the Eastern Cooperative Oncology Group established FAM as the regimen of first choice and showed a relatively low toxicity profile. $^{8}$
An overview of over 500 patients treated subsequently shows an overall response rate with FAM of $33 \%$ with a complete response rate of $<3 \%{ }^{3}$. Unfortunately, this initial response did not translate into an appreciable survival advantage and prompted the organisation of randomised trials in patients treated with FAM compared with 5-fluorouracil alone which have shown no survival difference and greater toxicity for FAM.' There are modifications of the FAM programme. In FAP, cisplatin is substituted for mitomycin-C, and a response rate of $50 \%$ was shown in a small study of 26 patients. ${ }^{10}$ Confirmatory studies are in progress.

In 1979, Cadman showed that prior treatment with methotrexate improved the efficacy of 5-fluorouracil by causing increased intracellular levels of 5-fluorouracil nucleotides, enhancing the inhibition of thymidylate synthetase. ${ }^{11}$ The results of this 'biochemical modulation' were reported in 1986 in a study of 100 patients - 59 of whom responded and 12 of whom had complete remissions. ${ }^{12}{ }^{13}$ These results have recently been confirmed in a prospective study and show the superiority of the methotrexate containing regimen, leading to its recommendation as the gold standard for all future gastric carcinoma trials. ${ }^{14}$

A regimen combining etoposide, adriamycin, and cisplatinum 'EAP' has been developed in Germany and applied to 145 patients with locally advanced disease. Altogether $57 \%$ responded and there were $20 \%$ complete remissions that extended to three years. ${ }^{15}$ The toxicity of EAP is considerable with grade 3-4 myelosuppression occurring in an unacceptably high proportion of patients. Because of the side effects, this treatment programme has been used only in young, fit individuals with locally advanced disease. The treatment is unlikely to benefit most gastric cancer patients, who are elderly and frail. The same group are investigating a similar combination designed specifically for use in patients with poor performance status, using etoposide, folinic acid, and 5-fluorouracil. Data are currently available for 51 patients showing a $51 \%$ response rate that included six complete responses. ${ }^{16}$

Attempts to improve the prospect of cure have been made by investigation of the advantages of neo-adjuvant chemotherapy. EAP has been evaluated in a small series of patients with locally advanced unresectable tumours. ${ }^{17}$ In 20 patients who underwent 'second look' laparotomy there was significant downstaging of disease in $10(50 \%)$ after two cycles of chemotherapy. These patients underwent complete resection of their tumours and had a median disease free interval of 16 months. 
In summary, chemotherapy is effective in a small proportion of patients with gastric cancer, but does not provide substantial improvements in the length or quality of life in most. Clinical trials continue with the aim of improving the prognosis for selected groups of medically fit individuals.

ASHA SAINI

Department of Clinical Oncology,

Royal Postgraduate Medical School,

Hammersmith Hospital,

Du Cane Road,

London W12 OHS

Correspondence to: Dr J Waxman

JONATHAN WAXMAN

1 Office of Population Censuses and Surveys Monitor. London: HMSO, 1986.

2 Douglas HO. Gastric cancer. Contemporary issues in clinical oncology. Edinburgh: Churchill-Livingstone, 1988.

3 MacDonald JS, Gohmann JJ. Chemotherapy of advanced gastric cancer. Present status, future prospects. Semin Oncol 1988; 15 (suppl 4): 42-9.

4 MacDonald JS, Schein PS, Wooley PV, et al. 5-fluorouracil, doxorubicin and mitomycin combination chemotherapy for advanced gastric cancer. Ann

5 Gastrointestinal tumour study group. Phase 2-3 chemotherapy studies in advanced gastric cancer. Cancer Treat Reports 1979; 63: 1871-6.

6 Gastrointestinal tumour study group. A comparative clinical assessment of combination chemotherapy in management of advanced gastric carcinoma. Cancer 1982; 49: 1362-6.
7 Gastrointestinal tumour study group. Randomised study of combination chemotherapy, in unresectable gastric cancer. Cancer 1984; 53: 13-7.

8 Douglass H, Lavin P, Goudsmit A, et al. An Eastern Co-operative Oncology Group evaluation of combinations of methyl CCNU, mitomycin C, adriamycin and 5-fluorouracil in advanced measurable gastric cancer. $\mathcal{F}$ Clin Oncol 1984; 2: 1372-81.

9 Cullinan S, Moertel C, Fleming T, et al. A comparison of three chemotherapeutic regimens in the treatment of

10 Moertel C, Rubin J, O'Connell MJ, Schutt AJ, Wiend HS. A phase 2 study of combined 5FU, doxorubicin and cisplatin in the treatment of advanced upper gastrointestinal adenocarcinomas. $\mathcal{F}$ Clin Oncol 1986; 4: advanced

11 Cadman E, Heimer R, Davis L. Enhanced 5-FU nucleotide formation after methotrexate administration. Explanation for drug synergism. Science 1979; 205: 1135-7.

12 Klein HO, Wickramanayke PD, Farrkh GH. A combination protocol, FAMTX for treatment of metastasised stomach cancer [Abstract]. Proc Am Soc Clin Oncol 1986; 84: 86

13 Wils J, Bleiberg H, Dalesio O, et al. An EORTC gastrointestinal group evaluation of the combination of sequential methotrexate and 5-FU combined with adriamycin in advanced measurable gastric cancer. 7 Clin Oncol 1986; 4: 1799-803.

14 Wils JA, Klein HO, et al. Sequential high dose methotrexate and fluorouracil combined with doxorubicin - A step ahead in the treatment of advanced gastric cancer. A trial of the European Organisation for Research and Treatment of Cancer Gastro-Intestinal Tract Cooperative Group. $\mathcal{F}$ Clin Oncol 1991; 9: 827-31.

15 Preusser $\mathrm{P}$, Wilke $\mathrm{H}$, Achterrath W, et al. Advanced gastric carcinoma; a phase II study with etoposide $(\mathrm{E})$, adriamycin $(\mathrm{A})$ and split course cisplatin $(\mathrm{P})=$ II study with etoposide $(\mathrm{E})$, adriamycin $(\mathrm{A})$ and split course cisplatin $(\mathrm{P})$ EAP [Abstract]. Proc Am Soc Clin Oncol 1987; 6: 292.

16 Wilke H, Preusser P, Fink U, et al. New developments in the treatment of gastric carcinoma. Semin Oncol 1990; 17 (suppl 2): 61-70.

17 Wilke H, Preusser P, Fink U, et al. Preoperative chemotherapy in locally advanced and non-resectable gastric cancer. A phase II study with etoposide, doxorubicin and cisplatin. $\mathcal{F}$ Calin Oncol 1989; 7: 1318-26. 